\title{
50-kHz-rate Rayleigh and filtered Rayleigh scattering thermometry using a pulse-burst laser
}

\author{
Yedhu Krishna ${ }^{1}$, Ariff M. Mahuthannan ${ }^{2}$, Deanna A. Lacoste ${ }^{3}$, and \\ Gaetano Magnotti ${ }^{4}$ \\ Clean Combustion Research Center, Physical Science and Engineering Division, 4326-WS16, Building 5, \\ King Abdullah University of Science and Technology (KAUST), Thuwal 23955-6900, Saudi Arabia
}

A one-dimensional Rayleigh/filtered Rayleigh scattering system is developed using a pulse burst laser and it is used to demonstrate temperature measurements at $50 \mathrm{kHz}$ repetition rate. The system utilizes a CCD camera operated in the subframe burst gating mode for improved signal to noise ratio. This improvement in precision is verified by conducting temperature measurements in a laminar flame and comparing the results with that obtained using a highspeed CMOS camera. Two experiments are conducted to demonstrate the capability of the system. Firstly, 1D Rayleigh thermometry is conducted in a turbulent jet flame with Reynolds number 15200. Time-resolved temperature profiles and spatially resolved integral time scales are presented. After this, we demonstrate a 50-kHz-rate filtered Rayleigh scattering thermometry experiment using the pulse burst laser. In this experiment, the 1D temperature profiles of a turbulent flame propagating through a narrow rectangular channel with a variable height of approximately $2 \mathrm{~mm}$ is studied. The test facility is designed to study the flame quenching process in narrow channels and has unique measurement challenges due to the limited optical access and short flow duration. Measurements at three heights, ranging from quenching to no quenching conditions, are presented and the evolution of temperature profile is discussed.

\section{Nomenclature}

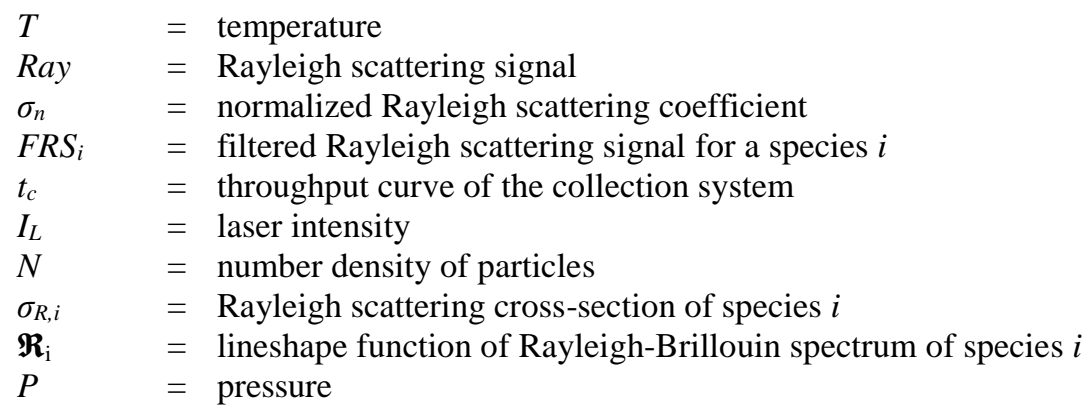

\footnotetext{
${ }^{1}$ Postdoctoral Fellow, Clean Combustion Research Center, King Abdullah University of Science and Technology, and AIAA Member.

${ }^{2} \mathrm{PhD}$ candidate, Clean Combustion Research Center, King Abdullah University of Science and Technology.

${ }^{3}$ Assistant professor, Clean Combustion Research Center, King Abdullah University of Science and Technology, and and AIAA Member.

${ }^{4}$ Assistant professor, Clean Combustion Research Center, King Abdullah University of Science and Technology, and AIAA Member.
} 


$\begin{array}{ll}u & =\text { velocity } \\ \theta & =\text { observation angle } \\ v & =\text { frequency } \\ v_{L} & =\text { laser frequency } \\ \boldsymbol{\Psi}_{\mathrm{I}} & =\text { transmission spectrum of the iodine cell } \\ P_{\text {cell }} & =\text { iodine cell pressure } \\ T_{\text {cell }} & =\text { iodine cell temperature }\end{array}$

\section{Introduction}

Several optical diagnostic techniques that require high laser pulse energies for achieving detectable signal levels have been extended to high repetition rates with the introduction of pulse-burst lasers capable of generating a burst of highenergy pulses [1, 2]. Temperature measurements using the Rayleigh scattering technique [3] have also been demonstrated at repetitions rates of the order of $10-100 \mathrm{kHz}[1,4-6]$ using such lasers. Many of the previous highspeed Rayleigh scattering systems used high-speed CMOS cameras as detectors $[5,7]$ and their noise performance acts as a limiting factor for achieving high-precision single-shot measurements. In addition to causing a low precision in Rayleigh measurements, this noise can also introduce a bias in the estimation of parameters such as thermal dissipation rate [8]. Several experimental and post-processing strategies have been used to minimize or correct for such adverse effects of noise [8,9]. Therefore, a system with improved signal-to-noise performance is always desirable.

Replacing the high-speed CMOS camera with a low-noise back-illuminated CCD camera improves the precision and increases the dynamic range of high-speed Rayleigh scattering systems. However, the slow read-out of CCD cameras, needed to achieve a low-readout noise, restrict the frame rates to very low values (tens of $\mathrm{Hz}$ for $1 \mathrm{D}$ measurements). This limitation can be circumvented by operating the camera in subframe burst gating (SBG) mode $[10,11]$. This mode allows the operation of low-noise CCD cameras at frame rates of up to 100000 frames per second without compromising the signal-to-noise ratio and the dynamic range [12].

In this paper, first we demonstrate a 50-kHz-rate one-dimensional Rayleigh scattering system that uses a CCD camera operated in the SBG mode. By direct comparison of one-dimensional temperature measurements obtained using this system in a laminar calibration flame with that obtained using a high-speed CMOS camera, we verify the improvement achieved in the instrument precision. Details of the experimental configuration and post-processing procedure are detailed. We then apply the system for measurements in a turbulent non-premixed jet flame and present the time-resolved temperature data, spatially resolved autocorrelation functions, and integral time scales in the probed region of the flame.

This approach is particularly relevant for experiments where the signal is weak and the precision is limited by the camera noise. Therefore, we applied the strategy to demonstrate another $50 \mathrm{kHz}$ experiment in which the signal is too low to be detected using a CMOS camera, but is within the detection limit of the CCD-based system. This is a filtered Rayleigh scattering (FRS) experiment to measure the time-resolved one-dimensional temperature profile of a turbulent flame propagating through a narrow rectangular channel of approximately $2 \mathrm{~mm}$ height within an enclosed facility. This test facility is a flame arrestor model that is designed to study the quenching phenomenon in flames propagating through narrow channels $[13,14]$. Such a measurement system would be relevant to several applications such as flame arrestors, micro-combustors, and prechambers of IC engines. A detailed understanding of transient quenching phenomena in channels would greatly benefit from these quantitative time-resolved temperature measurements. FRS imaging has previously been demonstrated at $\mathrm{MHz}$ repetition rate using a low energy pulse burst laser $(\sim 1 \mathrm{~mJ}$ per pulse at $532 \mathrm{~nm}$ ) [15]. FRS has also been extensively used for quantitative measurements in enclosed combustors, and in flames seeded with particles [16-20]. However, the test case presented here is a particularly challenging one due to proximity to the wall, and small collection angle. Moreover, obtaining time-resolved temperature profiles during the short span $(<1 \mathrm{~ms})$ of the flow requires a laser with a high repetition rate and an appropriate timing system. We overcome these challenges by using lasers bursts of temporally stretched pulses at a $50 \mathrm{kHz}$ repetition rate. To our knowledge, the experiment presented here is the first 1D FRS thermometry experiment using a high-energy pulse burst laser at a repetition rate as high as $50 \mathrm{kHz}$. This was achievable only due to the capability of the pulse burst system combining the pulse burst laser and the SBG mode of the CCD camera. This part of the work is a continuation and extension of our recent work [21] where we studied laminar flame propagation through such narrow channels by conducting FRS measurements at a $10 \mathrm{kHz}$ repetition rate. 


\section{Theory}

Rayleigh scattering is a widely used technique for temperature measurements in open atmospheric-pressure flames [3]. For a given pressure, the Rayleigh scattering signal from a gas sample is proportional to the number density of molecules and the Rayleigh scattering cross-sections of the species constituting the sample. For a gas mixture at atmospheric pressure, the total Rayleigh scattering signal of the gas sample can be approximated to be the molefraction-weighted sum of the Rayleigh scattering contributions from the different species. Therefore, if the mole fractions of various species in the sample are known, then the temperature in the targetted region can be obtained using the ideal gas law by comparing the Rayleigh scattering signal from the flame with the Rayleigh scattering signal obtained in a reference medium such as air. If $T_{\text {air }}$ is the temperature in air, Rayflame is the Rayleigh scattering signal in the flame, $\sigma_{n}$ is the normalized Rayleigh cross-section defined as the ratio of the effective Rayleigh cross-section of the sample mixture to that of air, then the flame temperature is given by the equation:

$$
T_{\text {flame }}=T_{\text {air }} \frac{\text { Ray }_{\text {air }}}{\text { Ray }_{\text {flame }}} \sigma_{n}
$$

Alternatively, a tailored gas mixture can be used such that the effective Rayleigh scattering cross-section does not change between air, reactants, and products [22]. In that case, $\sigma_{n}$ becomes $\sim 1$ and the temperature becomes inversely proportional to the Rayleigh scattering signal. This tailored-mixture approach is used for the Rayleigh scattering experiment in the current paper.

In the case of an enclosed combustor, the diffused surface scattering from the optical windows can cause large interference to the Rayleigh scattering signal. The Mie scattering from soot particles in flames as well as from droplets also has a similar effect. In most applications, these interferences are several orders of magnitude larger than the Rayleigh scattering signal from the atoms and molecules in the flame and compromise the accuracy of Rayleigh-based measurements $[23,24]$. The filtered Rayleigh scattering (FRS) technique overcomes this difficulty by absorbing the Mie/surface scattering components in the scattered light using a molecular filter [25, 26]. A wavelength-tunable seeded laser with a narrow linewidth is used as the light source and the laser wavelength is tuned to a strong absorption line of a molecular gas cell. In this experiment, a $532 \mathrm{~nm}$ laser is used as the light source and an iodine cell which has strong absorption features near this wavelength is used as the molecular filter. The surface/Mie scattering components have a narrow linewidth similar to that of the laser source and are absorbed. The Rayleigh-Brillouin scattering (RBS) signal is broader than the absorption lines of the cell and therefore, a portion of this signal is transmitted. This transmitted component of the RBS spectrum is the filtered Rayleigh scattering signal and can be used to estimate physical parameters such as temperature and velocity. The FRS signal scattered from a single species $i$ in a gas mixture can be expressed by [17]:

$$
F R S_{i}=t_{c} I_{L} N \sigma_{R, i} \int_{v} \Re_{i}\left(P, T, \mathbf{u}, \theta, v_{L}, v\right) \Psi_{I}\left(P_{\text {cell }}, T_{\text {cell }}, v\right) d v
$$

where $I_{L}$ is the laser intensity, $N$ is the number density, $\sigma_{R, i}$ is the differential Rayleigh scattering cross-section, $v$ is the frequency which varies over the range of integration of the FRS signal and $t_{c}$ is a conversion factor that depends on the collection system. $\Psi_{I}$ is the transmission spectrum of the iodine cell and a function of its length, pressure $P_{\text {cell, }}$ and temperature $T_{\text {cell. }} \boldsymbol{R}_{\mathrm{i}}$ is the lineshape function of the Rayleigh-Brillouin scattering (RBS) and it depends on the sample pressure $P$, temperature $T$, velocity $\mathbf{u}$, observation angle $\theta$, laser frequency $v_{L}$, and gas properties such as its molecular mass and transport coefficients.

In our experiment, the ratio of this FRS signal from the flame and that from the unburnt fuel is used to estimate temperature based on a simulated calibration curve. This FRS simulation was conducted using the Tenti S6 model [27] and the iodine absorption spectrum obtained using the Forkey model [28]. The RBS spectrum simulated using the Tenti S6 model was multiplied with the transmission spectrum of the iodine cell to obtain the FRS spectrum, which is then integrated in the frequency space to simulate an integrated FRS signal. Mole fraction was assumed to follow the temperature based on a counterflow simulation with the same reactant composition as used in the current experiment on one side and the product composition on the other side. The details regarding this simulation and the error analysis based on various counterflow simulation parameters can be found in [21]. This simulation is repeated for all the gas compositions (and corresponding temperatures) between the reactants and products based on the counterflow flame data. A Doppler shift was applied to the laser wavelength corresponding to the simulation of the flow FRS signal, based on the flow velocities observed in the experiment. The simulated FRS signals are normalized with FRS signal simulated for the reference gas mixture (unburnt fuel in our case) to obtain the calibration curve between temperature and reference-normalized FRS signal. This calibration curve is then used to convert the 
reference-normalized FRS signal obtained in the experiment to temperature. A direct simultaneous measurement of mole fraction using a technique such Raman spectroscopy $[18,29]$ would have been better, but this was not possible with the energy levels achievable using the current system and allowed by the damage threshold of the test facility windows.

\section{Experimental Configuration}

The experimental configurations of the Rayleigh scattering and filtered Rayleigh scattering (FRS) experiments are described below. The basic layouts of both the configurations are similar, except that the FRS set up additionally consists of a molecular absorption filter and a wavelength-monitoring unit.

\section{A. Rayleigh scattering experiment}

Rayleigh scattering thermometry experiments are conducted in a laminar flat-flame burner and a turbulent nonpremixed jet flame. The flat-flame burner uses a premixed mixture of methane and air in the ratio 0.09:1 by volume, surrounded by a co-flow of nitrogen. The turbulent jet flame burner uses a tailored mixture of $22.3 \% \mathrm{CH} 4,33.4 \%$ $\mathrm{H} 2$, and $44.7 \% \mathrm{~N} 2$, such that the Rayleigh cross-section remains almost constant in the probed region [22]. This allows direct temperature measurement from the Rayleigh signal. The fuel jet flows through a tube of inner diameter $4.5 \mathrm{~mm}$ and it is surrounded by a coflow of air. The Reynolds number is 15200 and measurements are conducted at a height of $10 \mathrm{~mm}$ from the fuel tube exit.

Figure 1 shows the schematic of the experimental set-up for the $50 \mathrm{kHz}$ Rayleigh scattering measurements. The laser source is a Spectral Energies QuasiModo 1200 seeded pulse-burst laser and the camera is a Princeton instrument PROEM-HS high-speed EMCCD camera with a 1024x1024 chip resolution. The second harmonic output from this pulse-burst laser is focused using a $500 \mathrm{~mm}$ lens to the flame. The laser is operated at $50 \mathrm{kHz}$ using a pulsed nanosecond seed, producing a burst of approximately 500 pulses, each with $\sim 100 \mathrm{~mJ}$ energy. No optical breakdown is observed at these conditions. The number of pulses is limited by the laser's burst length of $10 \mathrm{~ms}$. Relative shot-toshot variation in laser energy is monitored using a photodiode that collects light scattered from one of the mirrors.

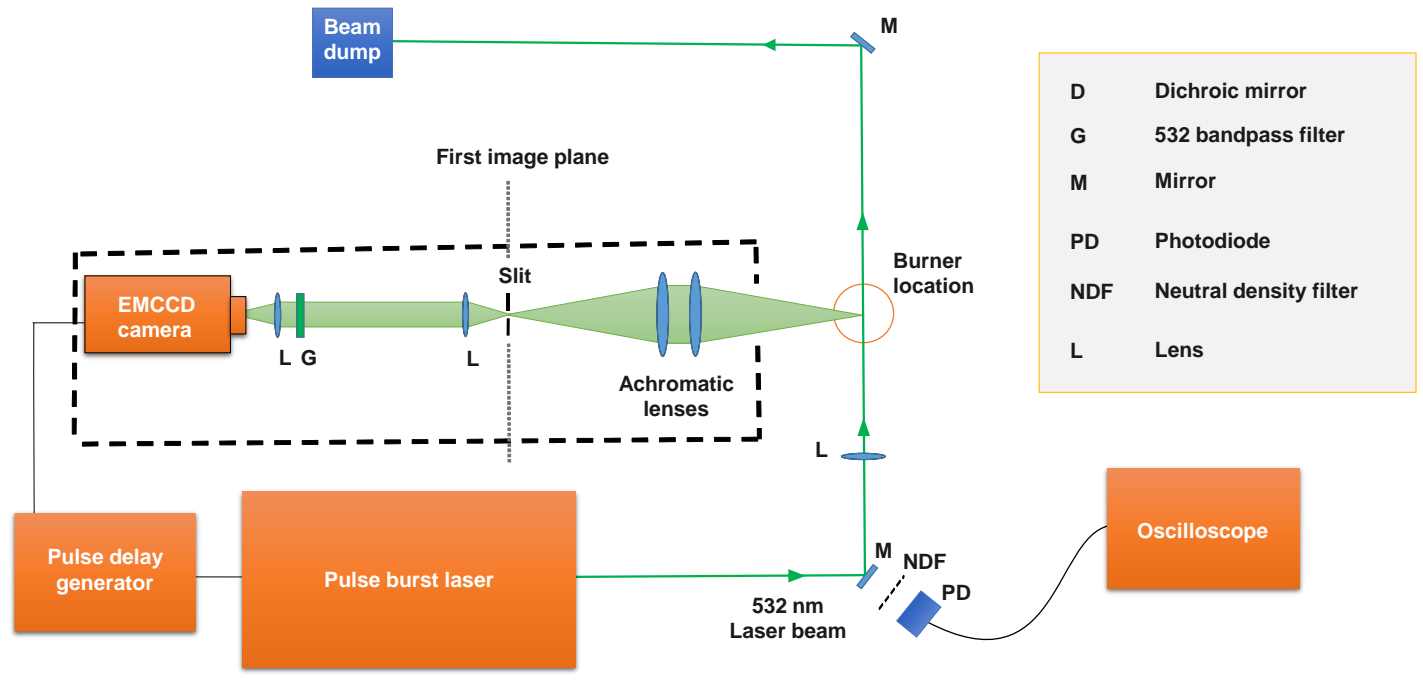

Fig. 1. Schematic diagram of the Rayleigh scattering experimental setup.

A pair of achromatic lenses with a diameter of $100 \mathrm{~mm}$ and focal lengths of $400 \mathrm{~mm}$ and $300 \mathrm{~mm}$ are used to collect light from the target flame and create an image of the scattered laser light on the other side of the lens pair. An adjustable rectangular slit is placed at this 'first image plane' to block light outside the region of the laser beam and to enable masking for subframe burst gating [11]. The light passing through the slit is further collected and collimated by a multi-element Nikon lens of focal length $200 \mathrm{~mm}$ and an f-number of 2.8, passed through a 532-nm bandpass filter with a full-width at half-maximum (FWHM) of 10nm and optical density of 4, and imaged on the EMCCD camera with a multi-element Nikon lens of $85 \mathrm{~mm}$ focal length and f-number 1.8. Even though the camera is an 
electron-multiplying CCD camera, it is operated in the low-noise CCD mode which provides the best signal-to-noise performance when sufficient signal is available.

In the subframe burst gating mode of operation, only $\mathrm{N}$ pixel rows of the camera chip are exposed for a given exposure time and the remaining portion is masked. After each exposure period, the charge is vertically shifted immediately towards the frame-transfer area. The frame rate is then determined by the shift-rate (Eg: 1.2 $\mu \mathrm{s} / \mathrm{row}$ ). After the entire chip is filled with the signal, the full-image is slowly read-out preserving the low-noise characteristic of the back-illuminated CCD camera. The number of subframes is decided by the size of the chip. The approach is particularly suitable for 1D imaging with a pulse-burst laser because these lasers are also limited in the number of pulses they can generate. Two variations of subframe burst gating, namely, Kinetics and Spectra Kinetics mode, are available on the camera. These two schemes are schematically shown in Fig. 2. In the kinetic mode of operation, a window of $n$ pixel rows farthest from the serial register is exposed. The number of subframes acquired in this mode is decided by the window height $n$. With a resolution of 1024 X 1024 and an equally big factory-masked frame transfer region, the total number of subframes that can be acquired during a single burst is [2048/n]. In the Spectra kinetics mode of operation, a window of $n$ pixels closest to the frame transfer region is exposed. Hardware binning is automatically conducted in the vertical direction before shifting the signal to the frame transfer region. This hardware binning capability is a major advantage of CCD cameras compared to CMOS cameras since it provides an improved noise reduction compared to software binning. Since the $n$ pixel rows are combined to a single row by binning, the number of subframes in the Spectra kinetics mode is not limited by the exposed window height, unlike in the Kinetics mode of operation. In our Rayleigh scattering experiment, we use the Spectra kinetics mode with a window height of 13 pixels and a shift rate of $1.2 \mu \mathrm{s}$ per pixel, which allows the camera to operate at $50 \mathrm{kHz}$ and acquire 1037 subframes. However, due to the limited burst length (number of pulses during the $10 \mathrm{~ms}$ burst duration) of the laser, only around 500 out of 1037 subframes are useful.

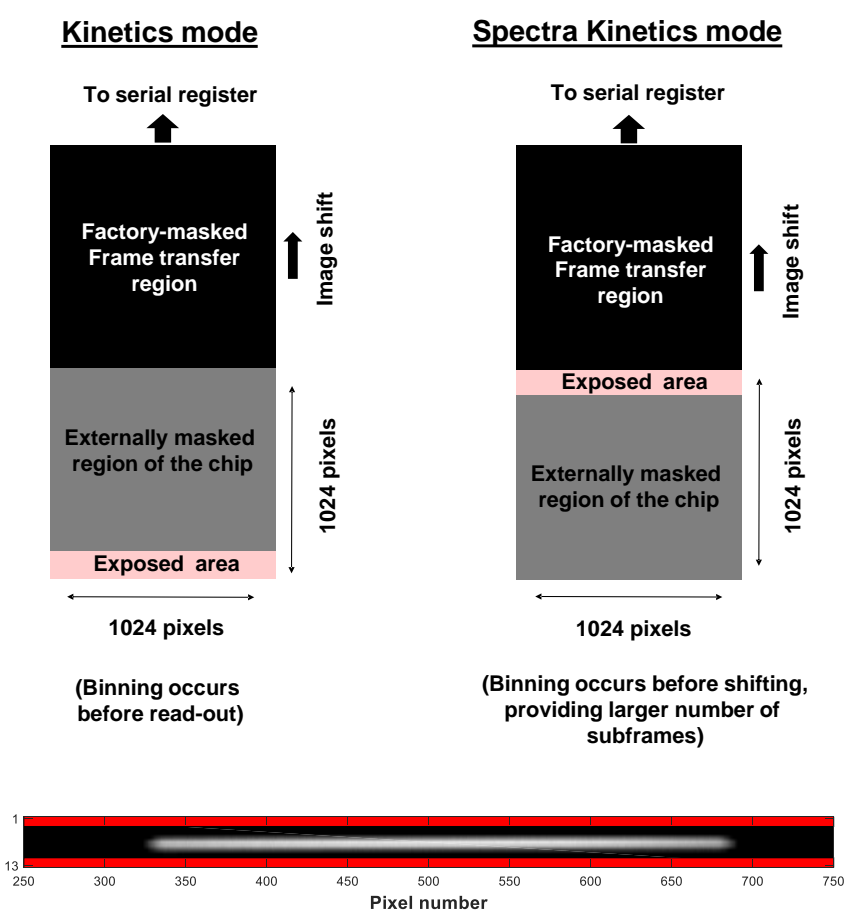

Fig. 2. Subframe burst gating: Kinetics vs Spectra kinetics mode in the camera (top) and the location of the laser beam relative to the region covered by the slit (marked in red) in the exposed window of the chip (bottom).

\section{B. Filtered Rayleigh scattering experiment}

The objective of this high-repetition-rate filtered Rayleigh scattering experiment is to measure the time-resolved temperature profiles of a turbulent flame propagating through a narrow rectangular channel that simulates a flame arrestor. A schematic representation of the laser beam path and the flame arrester test facility is shown in Fig. 3. The test facility consists of a chamber with two sections connected using a narrow rectangular channel and filled with premixed methane-air at an equivalence ratio of 0.8 . The flame is ignited in one chamber and it propagates to the 
second chamber through the narrow rectangular quenching channel. The test facility is designed such that the height of the quenching channel as well as the flow velocity can be varied. In this work, we used three channel heights: $2.4 \mathrm{~mm}, 2 \mathrm{~mm}$, and $1.7 \mathrm{~mm}$. Additional details of the facility can be found in [13].

Figure 4 shows the experimental configuration for the filtered Rayleigh scattering experiment. The laser and the camera are the same as those used in the Rayleigh scattering experiment. A $1000 \mathrm{~mm}$ focusing lens is used to focus the laser beam to the channel and is aligned to position the beam equidistant and parallel to the surfaces. To increase the collection angle, the beam location is laterally shifted by $1.5 \mathrm{~cm}$ away from the center of the channel and towards the collection optics. This location is sufficiently far from the side windows, so sidewall effects on the flow can be neglected. A laser with a narrow linewidth and tunable wavelength is required to implement filtered Rayleigh scattering. Therefore, the laser is operated with an external cavity diode laser (ECDL) as the seed. In order to avoid damage to the test facility windows and to avoid unwanted ignition of the premixed fuel, a temporally stretched pulse with a full width at half maximum of $200 \mathrm{~ns}$ and pulse energy of $\sim 20 \mathrm{~mJ}$ is used.
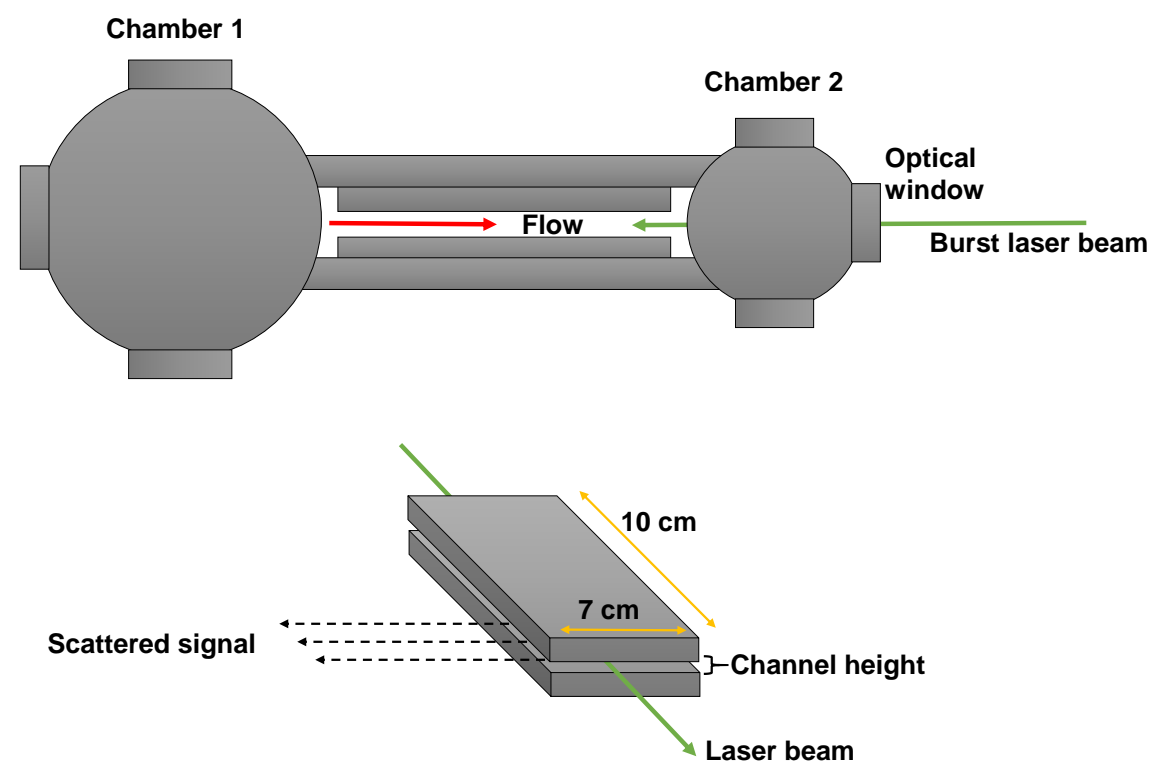

Fig. 3. Schematic representation of the narrow channel flow and the laser beam direction in the test facility.

The scattered signal emitted from within the channel is collected using a Nikkor lens of focal length $50 \mathrm{~mm}$ and $\mathrm{f}$ number of 1.2, placed $\sim 36 \mathrm{~cm}$ away from the probed region, and imaged to a focal plane. A slit placed at this focal plane acts as the mask for enabling the subframe burst gating. The signal transmitted through the slit is collimated using a Nikkor lens of $85 \mathrm{~mm}$ focal length and f-number 1.8, passed through a $532 \mathrm{~nm}$ bandpass filter with $10 \mathrm{~nm}$ FWHM, an iodine cell of length $25 \mathrm{~mm}$, and focused on the EMCCD camera using another Nikkor lens of $85 \mathrm{~mm}$ focal length and f-number 1.8. The entire setup is enclosed and the experiment is conducted with room lights off to avoid interference from room light.

The wavelength of the seeded pulse-burst laser is tuned to one of the strong absorption lines (P $83 \mathrm{v} 0-33$ ) of iodine. The cell is maintained at a temperature of $65^{\circ} \mathrm{C}$ to avoid condensation on the cell walls and to ensure strong, stable absorption. The peak absorbance of the iodine was characterized separately before the experiment by sampling a portion of the laser beam and passing through the cell. The maximum reduction in the transmitted signal, i.e. transmission corresponding to the absorption peak, provides the maximum suppression of Mie/surface scattering possible with the system. This is approximately 3000 for our experiment and is limited by the spectral purity [30] of the laser which was found to be 0.9995 based on the experimentally obtained absorption spectrum. This suppression level is deemed to be sufficient to completely suppress the Mie/surface scattering component as verified by the measurements taken after evacuating the test cell. A wavelength meter is used to tune the laser near the desired iodine absorption line, and further fine-tuning of laser wavelength is done using a wavelength-monitoring setup that consists of a 5-inch iodine cell (reference iodine cell), as explained below. A portion of the laser beam is sampled and split into two parts: one of the beams passes through the reference iodine cell and is detected using a photodiode, and the other beam is directly detected using a second photodiode. The ratio of the two photodiode signals follows the 
transmission spectrum of the iodine cell, and it is calibrated against the laser wavelength using the wavelength meter. By monitoring this photodiode ratio and making sure that the ratio remains near the minimum value, the uncertainties due to the wavelength drift of the laser are minimized during the experiment.

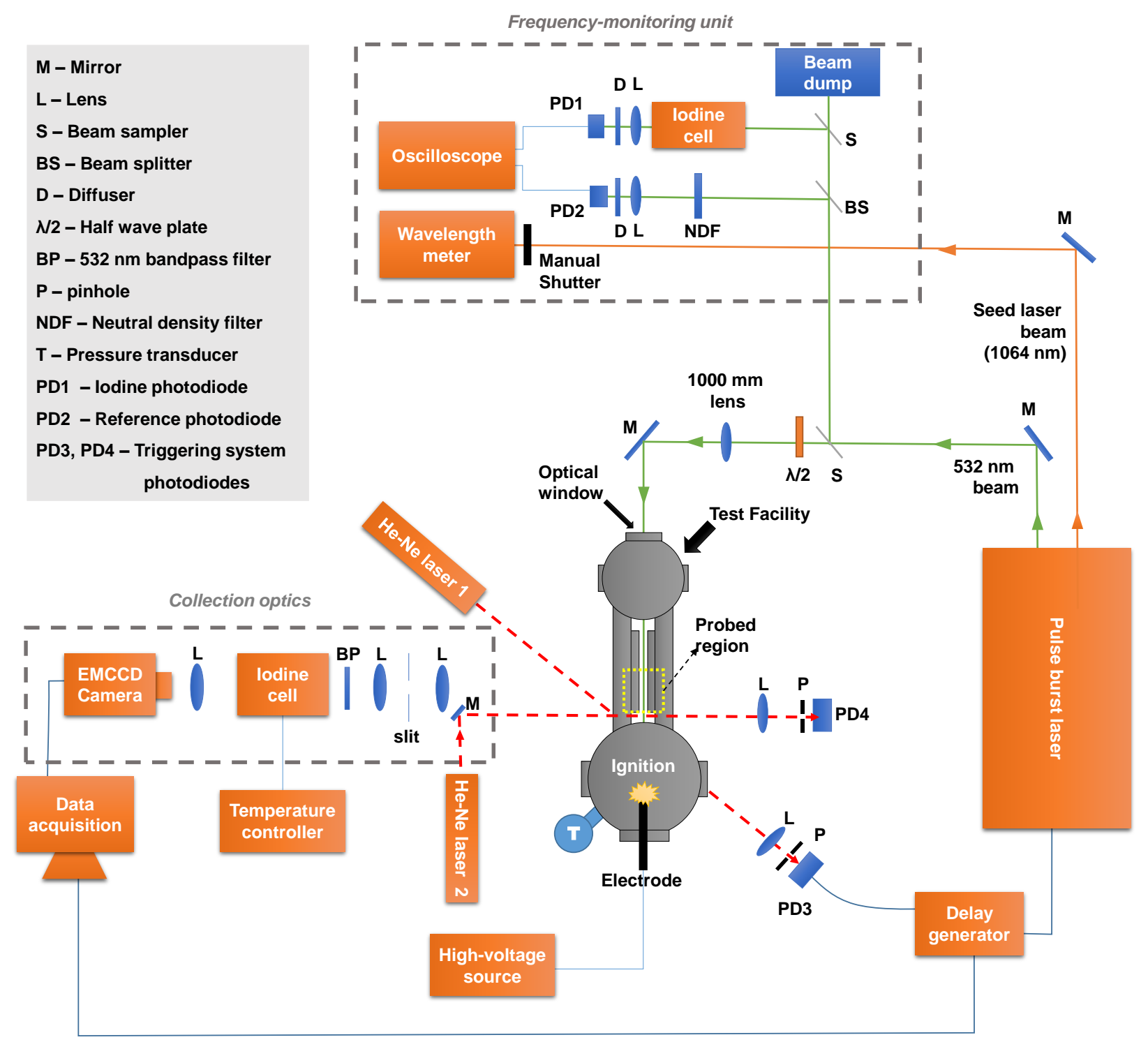

Fig. 4. Schematic diagram of the FRS experimental setup.

The Kinetics mode of operation with a window height of 16 pixels, a shift rate of $1.2 \mu \mathrm{s} / \mathrm{row}$ and vertical hardware binning option was used on the camera to achieve a $50 \mathrm{kHz}$ repetition rate. Because the hardware binning in Kinetics mode occurs only after the entire shifting process is complete and just before readout, the Kinetics mode was slightly faster compared to the Spectra kinetics option and allowed to approach $50 \mathrm{kHz}$ repetition rate with a window height of 16 pixels and $1.2 \mu \mathrm{s} / \mathrm{row}$ shift rate, which was not possible using the Spectra kinetics option. As a result, the number of subframes was limited to 128, but this was more than enough because the flow velocity was around $80 \mathrm{~m} / \mathrm{s}$ and only about 20-30 frames are required to capture the time-resolved FRS profiles of the flow as the flame passes across the $40 \mathrm{~mm}$-long field of view.

The flow has a velocity of about $80 \mathrm{~m} / \mathrm{s}$ and the useful window of the flow duration is only less than a millisecond. A careful synchronization between ignition and data acquisition is required to capture the relevant period of the flow. For this, a He-Ne laser beam is passed through a location upstream of the quenching channel's entrance and directed to a photodiode PD3 mounted behind a pinhole. The beam is focused through the pinhole using a convex lens of 150 
$\mathrm{mm}$ to maximize the photodiode signal. When the flame reaches the location of the He-Ne laser beam after ignition in the first chamber, the beam is slightly deflected due to the density change, causing a drop in the photodiode signal. This signal drop is detected by a delay generator which then triggers the pulse burst laser and the camera. Another HeNe laser beam was passed through a location just upstream of the channel entrance and passed through a photodiode PD4 in the same manner to detect the time at which the flow enters the channel.

\section{Results and Discussion}

In this section, we discuss the experimental results from both the Rayleigh scattering experiment and the filtered Rayleigh scattering experiment separately.

\section{A. Rayleigh scattering experiment}

Firstly, Rayleigh scattering signals obtained in air and a laminar flame using the CCD-based system were compared against those obtained using a CMOS-based system, for direct comparison of their precision. For the CMOS-based test, the CCD camera in Fig. 1 was simply replaced with a high-speed CMOS camera (Lavision Imager pro-HS). The images are software binned by 6 pixels in the horizontal direction, background subtracted, and energy-normalized using the photodiode signal (Fig. 1). Then the flame signal is divided by the air Rayleigh signal obtained just before the experiment and the temperature is estimated based on Eq.1, where the reference air temperature is measured using a thermocouple. A variation in beam focusing was observed within every burst of the laser, the cause of which could be thermal focusing effects within the laser. This variation affects the amount of light collected by the photodiode used for energy normalization, thus reducing the precision. Therefore, normalization with the air Rayleigh signal was done on a pulse-to-pulse basis as follows. Air Rayleigh signal from 40 burst measurements in air was obtained before the flame experiment and averaged on a pulse-to-pulse basis, resulting in a 'mean air burst' signal. After the flame experiment, the flame Rayleigh signal from the ' $n$ 'th pulse in a burst was normalized with the air Rayleigh signal from the ' $n$ 'th pulse in the mean air burst. A similar procedure is followed for all the single-shot experiments using the pulse burst laser. The coefficient of variation (COV) in temperature, i.e., the standard deviation of 400 pulses, expressed as a fraction of the mean expressed as a percentage, is used to represent the instrument precision. The COV obtained using both the systems are shown in Fig. 5. It can be seen that the COV in air measurements using the CCDbased system is at least $50 \%$ better compared to that obtained using the CMOS-based system. In the flame, the improvement in COV is better than 2 times when we use the CCD-based system, verifying the advantage provided by the improved noise performance of CCD cameras.

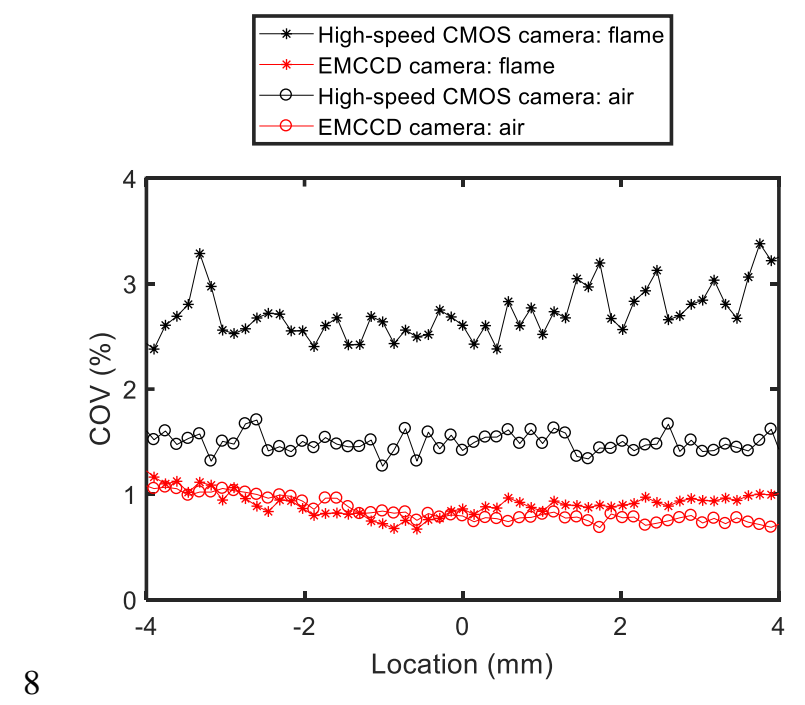

Figure 5. Comparison of the coefficient of variation in temperature measurements obtained using the CCDbased system and the CMOS camera.

After this, the Rayleigh scattering system is used to measure the temperature in the turbulent non-premixed jet flame, the details of which are described in Section IV.A. Measurements are conducted at a height of $10 \mathrm{~mm}$ from the 
exit of the burner. The flame is assumed to be symmetric and results only on one side of the flame axis are presented. Figure 6 shows the mean temperature profile and the corresponding standard deviation, and Fig. 7 shows the timeresolved temperature profiles at six radial locations, marked as A, B, C, D, E, and F in Fig. 6, during a randomly selected burst.

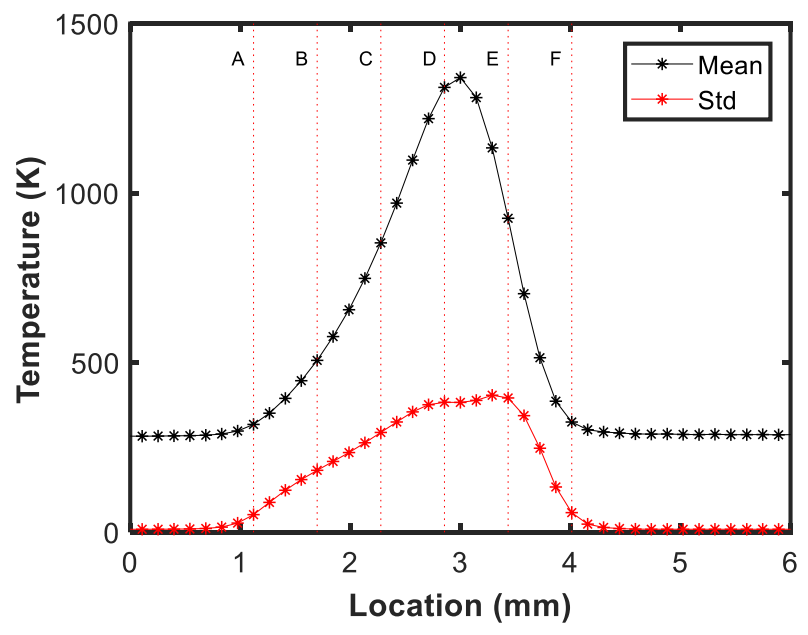

Figure 6. The mean radial temperature profile and standard deviation in the turbulent jet flame, at a height of $10 \mathrm{~mm}$ from the fuel tube exit.

The average temperature shows that the potential core extends to a radial distance of $\sim 0.8 \mathrm{~mm}$ and that the peak temperature occurs at a radial distance of $3 \mathrm{~mm}$. The temperature standard deviation increases with the radial distance on the fuel-side, and it peaks on the oxidizer side (location E), before rapidly dropping to a few $\mathrm{K}$ when reaching the laminar airflow. The temperature time series shown in Fig.7 for the selected location provides greater insight into the combustion process. On the fuel-side (A-C), the turbulent flow issuing from the tube leads to fast temperature fluctuations that are not well-resolved by the $50 \mathrm{kHz}$ measurements. Near the peak temperature (location D) the fast fluctuations observed closer to the jet axis, are coupled to a slower temperature evolution that is sufficiently resolved by the $50 \mathrm{kHz}$ measurements. At location $\mathrm{E}$, on the oxidizer side, where the standard deviation peaks, the highfrequency temperature fluctuations are no longer significant, and a smoother temporal evolution, properly resolved by the instrument is observed. The slow-temporal evolution of the temperature is indicative of the mixing between the laminar air-flow and the combustion products.
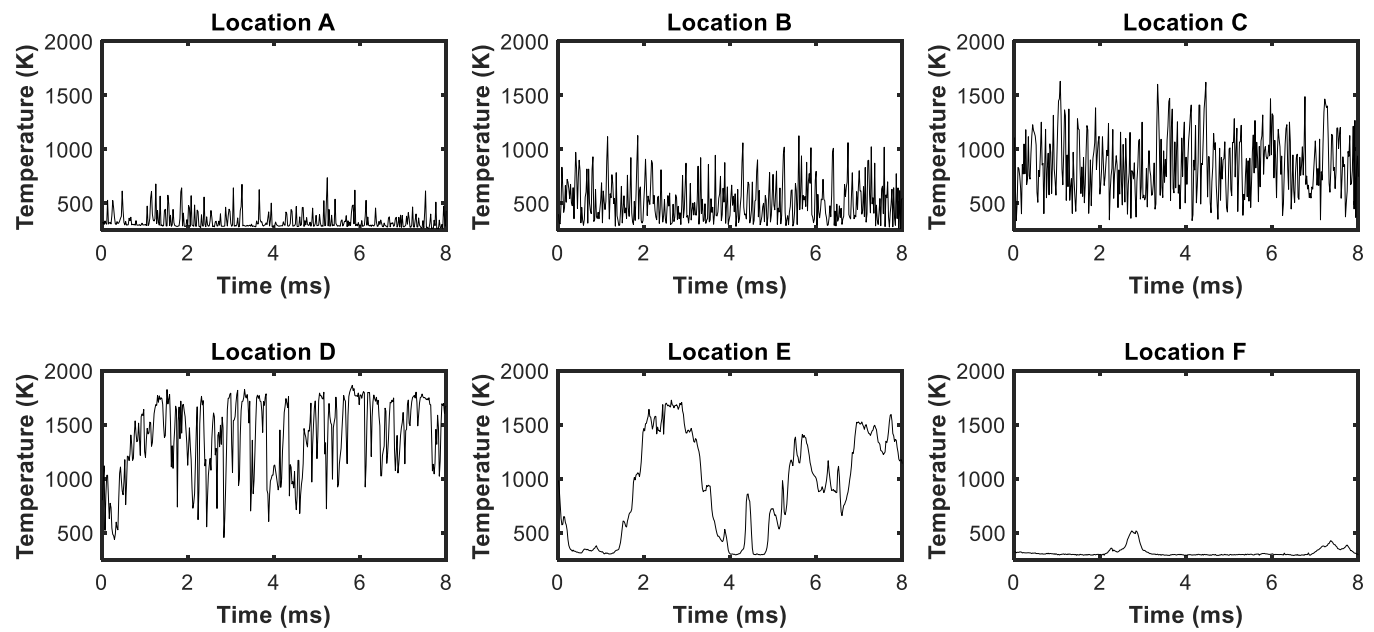

Figure 7. The time-resolved temperatures at six radial locations at a height of $10 \mathrm{~mm}$ from the fuel tube exit. 
The integral time scale estimated based on the autocorrelation function in the flame is an important parameter for studying turbulent flames. Integral time scales of $\mathrm{OH}$ and number density in non-premixed turbulent flames have been reported before $[8,31,32]$. The autocorrelation functions at the six locations marked in Fig. 6 are shown in Fig. 8. The integral time scales estimated based on the autocorrelation functions are plotted in Fig. 9 as a function of radial location. Only the region between $1 \mathrm{~mm}$ and $4 \mathrm{~mm}$ is shown since the flame exists only in this region. It can be seen that the integral time scales increases as we move from $\mathrm{r}=1 \mathrm{~mm}$ and reaches a maximum of $\sim 0.5 \mathrm{~ms}$ at $\mathrm{r}=3 \mathrm{~mm}$ within this region. This value is of the same order as that observed in the DLR-A flame, which is similar to the flame used in this study but has a larger fuel tube with an inner diameter of $8 \mathrm{~mm}$ [8]. The focus of the current work is only the demonstration of the capabilities of this 50-kHz-rate measurement system. A detailed study of the flame at various flame locations is beyond the scope of this paper.

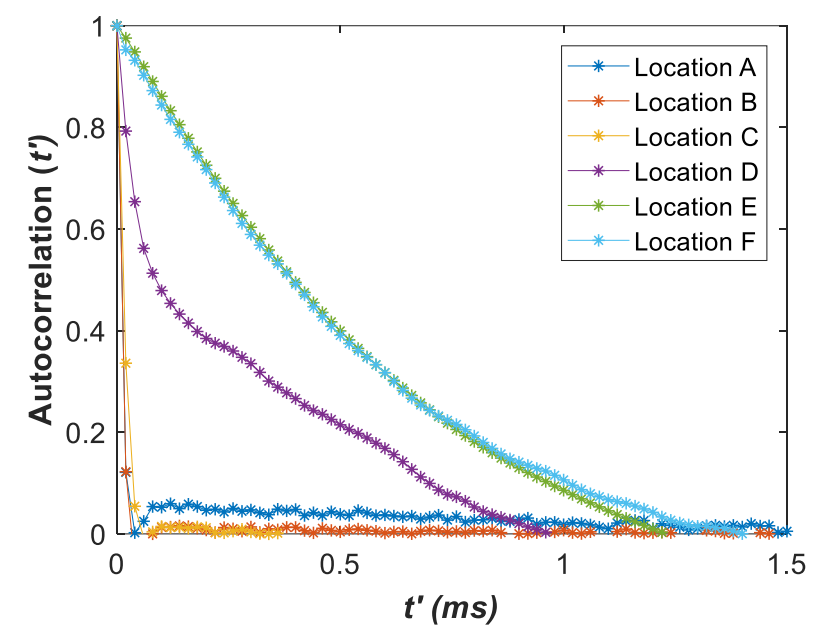

Figure 8. The autocorrelation curves at six radial locations at a height of $10 \mathrm{~mm}$ from the fuel tube exit.

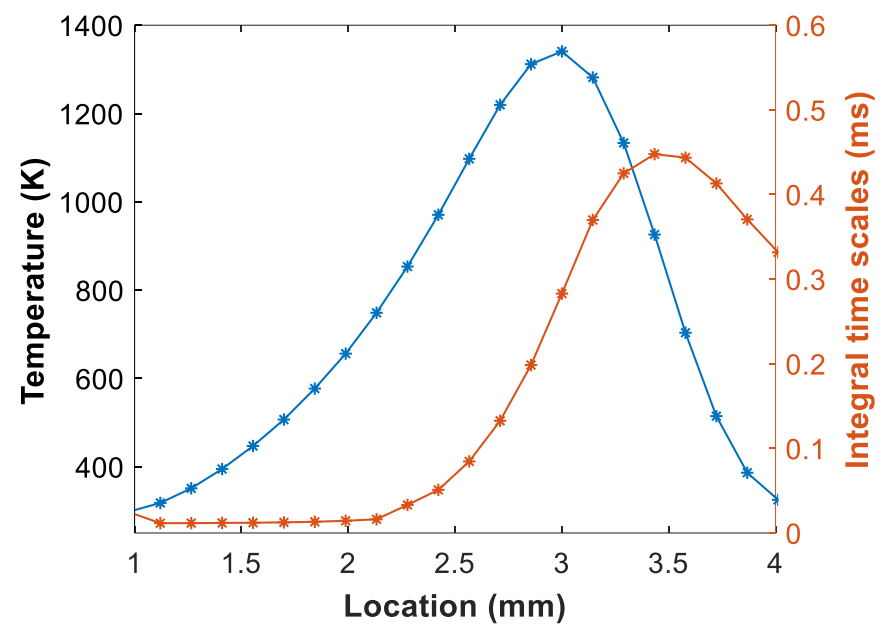

Figure 9. The integral time scale in the flame as a function of location.

\section{B. Filtered Rayleigh scattering experiment}

In the $50 \mathrm{kHz}$ filtered Rayleigh scattering experiment, time-resolved one-dimensional temperature measurements are demonstrated in the flame-arrestor model described in Section 2B. The measurement volume is $40 \mathrm{~mm}$ and starts at the entrance of the narrow rectangular channel. The pixel resolution after software binning by 2 in the horizontal direction is $170 \mu \mathrm{m}$. Measurements were conducted for three different channel heights $-2.4 \mathrm{~mm}, 2 \mathrm{~mm}$, and $1.7 \mathrm{~mm}$. Fig. 10 presents a comparison between the pressure traces of the quenching and no-quenching cases. When flame quenching occurs, the flame does not reach the second chamber, resulting in a lower pressure rise, providing a simple 
criterion to distinguish between quenching and no-quenching cases. For the experiments reported here, flame quenching occurred only for the channel height of $1.7 \mathrm{~mm}$. Preliminary tests showed that, due to the limited optical access, the resulting signal level was too low to be detected using an unintensified high-speed CMOS camera, making this an excellent test case for the subframe burst approach using the CCD camera.

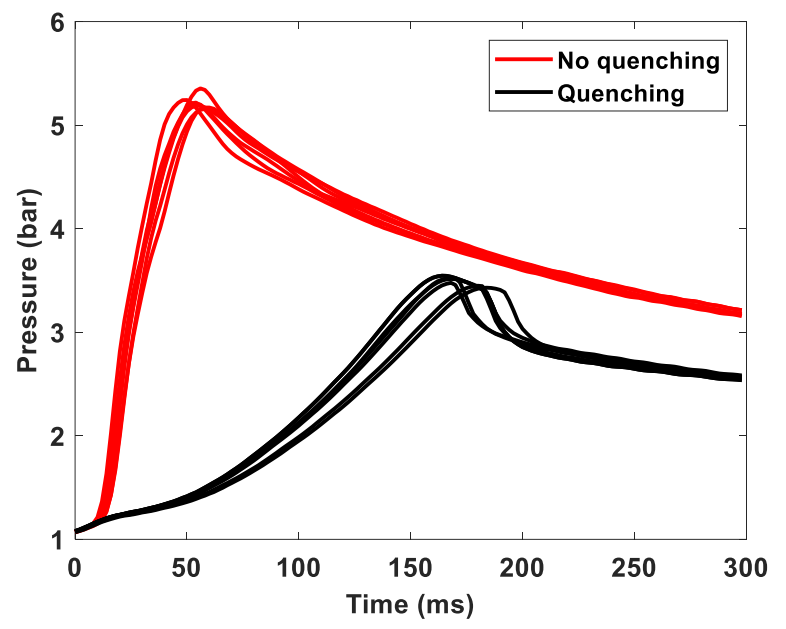

Figure 10. Comparison between pressure profiles observed for quenching and no-quenching test runs.

The measured 1D FRS signal was de-noised with a wavelet adaptive thresholding and reconstruction algorithm (WATR) [33]. The algorithm has been validated for 1D Raman and Rayleigh measurements using CCD cameras and can be extended directly to the $\mathrm{kHz}$ measurements without any modifications [34]. The algorithm increases the signalto-noise ratio without introducing artificial smoothness, making it a suitable approach for the measurement of spatial gradients. After background subtraction and energy normalization, the signal is normalized by the FRS signal from the non-reacting fuel mixture on a pulse-to-pulse basis as explained in Section V.A to obtain the reference-normalized FRS signal. The calibration curve obtained using the simulation, as explained in Section III, is used to estimate the temperature based on this reference-normalized FRS signal. The single-shot precision of the system was estimated by taking measurements in air with a reduced laser energy such that the signal level matches the signal in the flame. The COV within a burst, between bursts, and overall COV were $2.3 \%, 3 \%$, and $4.21 \%$, which improved to $0.95 \%, 3 \%$, and $3.6 \%$ after applying the WATR algorithm. The accuracy, estimated as the deviation of the average temperature measurement within a burst from the temperature measured by a thermocouple, is $<3 \%$, the same as that obtained in [21] based on measurements in a McKenna burner.
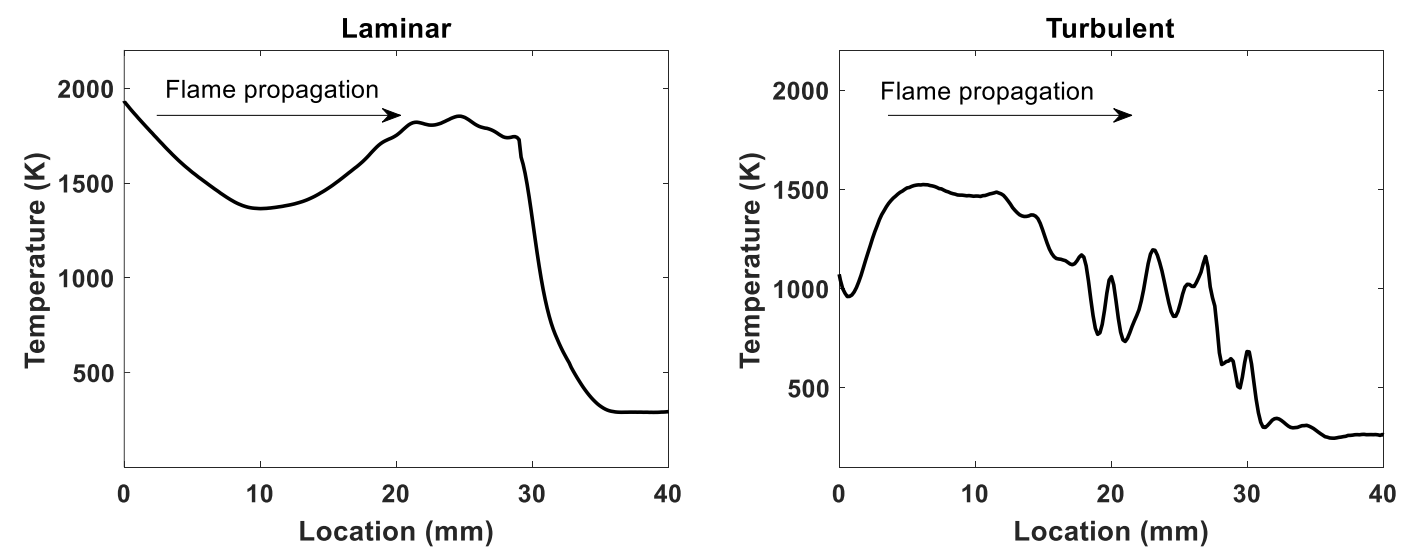

Figure 11. Comparison between temperature profiles of laminar and turbulent flows through the channel, for a channel height of $1.7 \mathrm{~mm}$. 
The temperature profiles obtained for a laminar flow case and a turbulent flow case at a channel height of $1.7 \mathrm{~mm}$ is shown in Fig. 11 for comparison. Contrary to the smoother profile obtained in our previous study on laminar flame propagation through such a channel [21], the 1D temperature profiles show large fluctuations revealing the turbulent nature of the process. Localized extinction and ignition events may occur, but this cannot be inferred from the temperature traces alone. Fig. 12 shows the time-resolved temperature data for the turbulent flow at three instants of time, for the three channel heights studied. The trends in temperature profiles are quite different between the different channel heights, but were quite repeatable for a given channel height. This was verified by repeating the experiments at least 6 times at each channel height.
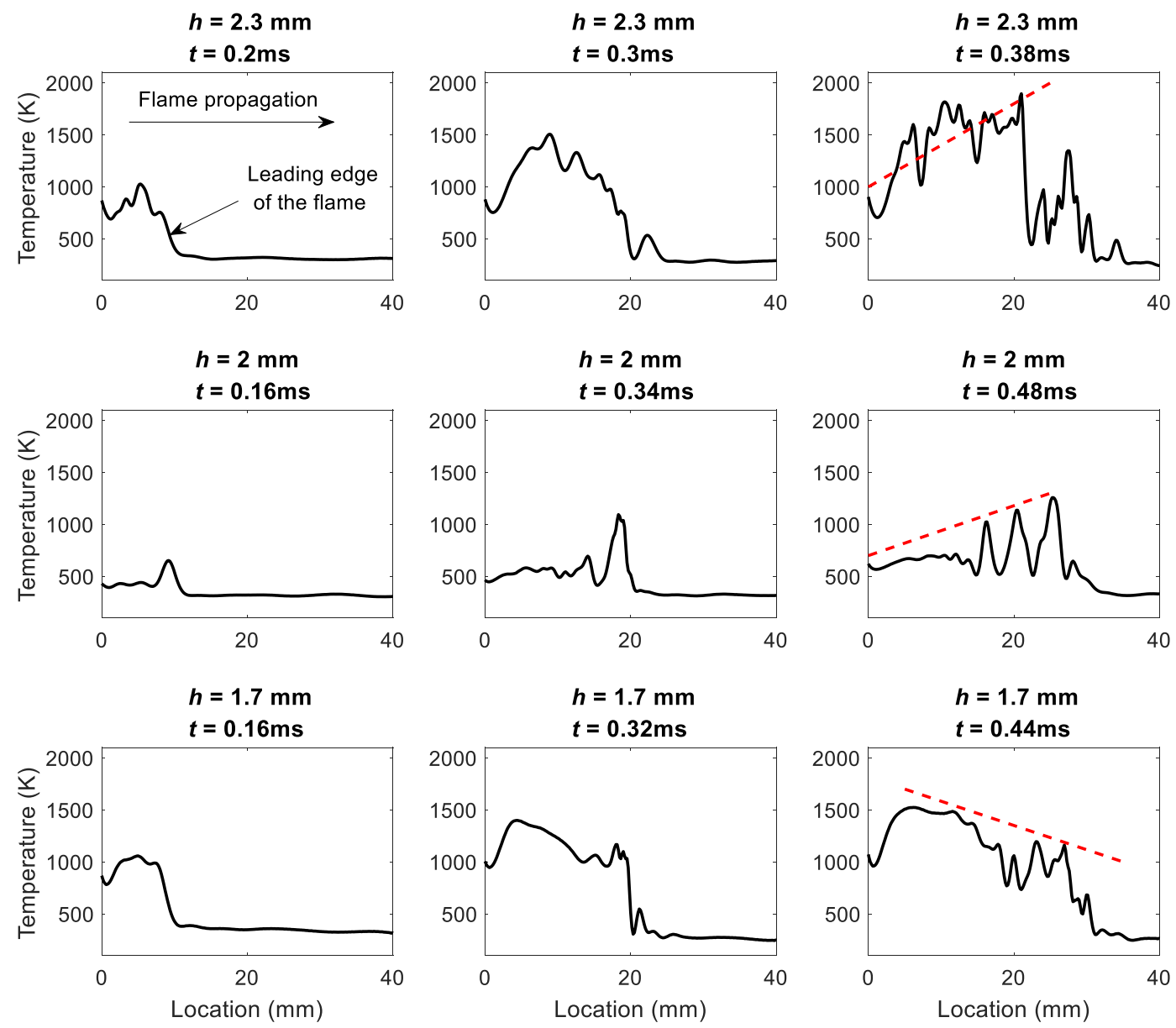

Figure 12. 1D temperature profiles in the channel at three consecutive instants of flame propagation, for three channel heights. $t=0 \mathrm{~ms}$ corresponds to the time at which the flame enters the channel.

For the channel height of $2.3 \mathrm{~mm}$, the temperature is around $800 \mathrm{~K}$ after it enters the channel and it gradually increases to around $2000 \mathrm{~K}$ as the flame reaches the location $30 \mathrm{~mm}$ downstream of the entrance. For the channel height with $2 \mathrm{~cm}$ height, the flow temperature seems to be close to the reactant temperature until the flame starts to appear at a location about $10 \mathrm{~mm}$ downstream of the entrance. After about $0.3 \mathrm{~ms}$, distinct temperature peaks appear along the channels, followed by a drop to the reactant temperature. This is likely due to the three-dimensional nature of the flame, moving in and out of the probing plane. The flame does not quench at this height. A simultaneous Raman scattering experiment would have provided more information about the process, but this was not achievable using the current system because of the limited optical access and energy levels. For the channel height of $1.7 \mathrm{~mm}$, the flame starts with a temperature of about $800 \mathrm{~K}$ and increases to a temperature above $1500 \mathrm{~K}$ in the first $10 \mathrm{~mm}$ downstream of the channel entrance, but then due to the increased heat loss at this channel height, the temperature gradually reduces 
after a location of $20 \mathrm{~mm}$ and eventually leads to flame quenching. The over-all slope of the envelope of peak temperatures as the flame develops across the channel provides an indication of the quenching event. For example, this slope, indicated with a red dashed line in Fig. 12, is positive for the first 2 cases where there is no quenching and negative for the last case (channel height $1.7 \mathrm{~mm}$ ) where quenching happens.

In our study on laminar flame propagation through the channel [21], we observed that the velocities for the quenching flow cases were lower compared to the no-quenching cases. One interpretation based on this observation was that the heat loss coefficient at the flow-wall interface can be assumed to be constant for all the cases and the heat loss at the lower channel heights is higher due to the longer residence time of the flow at these smaller velocities. In the current work, the velocity at each channel height is estimated based on the time taken by the leading edge of the flame, as indicated in the first subplot of Fig. 12, to travel from the entrance of the channel to the location $40 \mathrm{~mm}$ downstream of it. The average velocity in the first $40 \mathrm{~mm}$ of the channel was $92 \pm 7 \mathrm{~m} / \mathrm{s}$ for the channel height 2.3 $\mathrm{mm}$ and $78 \pm 3 \mathrm{~m} / \mathrm{s}$ for the channel height $1.7 \mathrm{~mm}$. However, for the channel height of $2 \mathrm{~mm}$, the velocity was found to be lower: $65 \pm 3 \mathrm{~m} / \mathrm{s}$. As mentioned earlier, the flame development is quite different for this channel height, compared to the other two cases. More experiments coupling with other techniques are required for a better understanding of the process. For example, since the behavior of flame is found to be quite repetitive at a given height, 1D or 2D OH laser-induced fluorescence experiments at various lateral locations of the flow would provide an idea about the 3D structure of the reaction zone, and would throw light into the two-dimensional or three-dimensional effects involved.

\section{Conclusion}

A high-repetition-rate diagnostic system that combines a high-energy pulse burst laser and subframe burst mode operation of a CCD camera was developed for one-dimensional flame temperature measurements. A Rayleigh scattering experiment at $50 \mathrm{kHz}$ repetition rate, conducted in a laminar flame verified that the single-shot precision of the system improved by at least 2 times compared to the measurements based on a high-speed CMOS camera. Timeresolved one-dimensional temperature measurements were then conducted in a turbulent jet flame, and the ability of the system to measure the autocorrelation functions and to estimate the integral time scales at various locations of the flame was demonstrated. After this, a 50-kHz-rate filtered Rayleigh scattering experiment in a narrow rectangular channel, where the signal was too low to be detected using the CMOS camera, was demonstrated by modifying the high-speed system to include a molecular absorption filter and a wavelength-monitoring system. Measurements were conducted in a turbulent flame propagating through this channel, for three different channel heights. The velocity of the flow was measured by tracking the flame front. Temperature profiles in the flow as the flame traveled across a distance of $40 \mathrm{~mm}$ from the entrance of the channel were quite repetitive for a given height but varied between channel heights. Large fluctuations were observed in temperature, indicative of pockets of reactants or a projection of the 3D flow in the line of the laser. The over-all slope of the envelope of peak temperatures was predictive of the quenching event. The results showed the necessity to couple these FRS measurements with other techniques such as Raman spectroscopy and laser-induced fluorescence for a better understanding of the flame structure.

\section{Acknowledgments}

This work was supported by funding from King Abdullah University of Science and Technology.

\section{References}

[1] Fuest, F., Papageorge, M. J., Lempert, W. R., and Sutton, J. A. "Ultrahigh laser pulse energy and power generation at $10 \mathrm{kHz}, "$ Optics Letters Vol. 37, No. 15, 2012, pp. 3231-3233. doi: 10.1364/OL.37.003231

[2] Slipchenko, M. N., Miller, J. D., Roy, S., Gord, J. R., Danczyk, S. A., and Meyer, T. R. "Quasi-continuous burstmode laser for high-speed planar imaging," Optics Letters Vol. 37, No. 8, 2012, pp. 1346-1348.

doi: 10.1364/OL.37.001346

[3] Miles, R. B., Lempert, W. R., and Forkey, J. N. "Laser Rayleigh scattering," Measurement Science and Technology Vol. 12, No. 5, 2001, pp. R33-R51.

doi: 10.1088/0957-0233/12/5/201

[4] Jiang, N., Hsu, P. S., Mance, J. G., Wu, Y., Gragston, M., Zhang, Z., Miller, J. D., Gord, J. R., and Roy, S. "Highspeed 2D Raman imaging at elevated pressures," Optics Letters Vol. 42, No. 18, 2017, pp. 3678-3681.

doi: 10.1364/OL.42.003678 
[5] Patton, R. A., Gabet, K. N., Jiang, N., Lempert, W. R., and Sutton, J. A. "Multi-kHz mixture fraction imaging in turbulent jets using planar Rayleigh scattering," Applied Physics B Vol. 106, No. 2, 2012, pp. 457-471. doi: 10.1007/s00340-011-4658-1

[6] Grib, S. W., Jiang, N., Hsu, P. S., Danehy, P. M., and Roy, S. "Rayleigh-scattering-based two-dimensional temperature measurement at 100-kHz frequency in a reacting flow," Optics Express Vol. 27, No. 20, 2019, pp. 27902-27916. doi: 10.1364/OE.27.027902

[7] Papageorge, M. J., McManus, T. A., Fuest, F., and Sutton, J. A. "Recent advances in high-speed planar Rayleigh scattering in turbulent jets and flames: increased record lengths, acquisition rates, and image quality," Applied Physics B Vol. 115, No. 2, 2014, pp. 197-213.

doi: 10.1007/s00340-013-5591-2

[8] Wang, G. H., Clemens, N. T., Varghese, P. L., and Barlow, R. S. "Turbulent time scales in a nonpremixed turbulent jet flame by using high-repetition rate thermometry," Combustion and Flame Vol. 152, No. 3, 2008, pp. 317-335. doi: https://doi.org/10.1016/j.combustflame.2007.08.010

[9] Wang, G. H., Clemens, N. T., and Varghese, P. L. "Two-point, high-repetition-rate Rayleigh thermometry in flames: techniques to correct for apparent dissipation induced by noise," Applied Optics Vol. 44, No. 31, 2005, pp. 6741-6751. doi: $10.1364 / A O .44 .006741$

[10] Kojima, J., Fischer, D., and Nguyen, Q.-V. "Subframe burst gating for Raman spectroscopy in combustion," Optics Letters Vol. 35, No. 9, 2010, pp. 1323-1325. doi: 10.1364/OL.35.001323

[11] Krishna, Y., Tang, H., Elbaz, A. M., and Magnotti, G. "High-speed Rayleigh-Raman measurements with subframe burst gating," Optics Letters Vol. 44, No. 17, 2019, pp. 4091-4094. doi: 10.1364/OL.44.004091

[12] Roy, S., Hsu, P. S., Jiang, N., Slipchenko, M. N., and Gord, J. R. "100-kHz-rate gas-phase thermometry using 100-ps pulses from a burst-mode laser," Optics Letters Vol. 40, No. 21, 2015, pp. 5125-5128. doi: $10.1364 /$ OL. 40.005125

[13] Mahuthannan, A. M., Damazo, J. S., Kwon, E., Roberts, W. L., and Lacoste, D. A. "Effect of propagation speed on the quenching of methane, propane and ethylene premixed flames between parallel flat plates," Fuel Vol. 256, 2019, p. 115870. doi: https://doi.org/10.1016/j.fuel.2019.115870

[14] Mahuthannan, A. M., Lacoste, D. A., Damazo, J., Kwon, E., and Roberts, W. L. "Flame Quenching Dynamics of High Velocity Flames in Rectangular Cross-section Channels," 55th AIAA Aerospace Sciences Meeting. American Institute of Aeronautics and Astronautics, 2017.

[15] Lempert, W., Wu, P. F., Miles, R., Lempert, W., Wu, P. F., and Miles, R. "Filtered Rayleigh scattering measurements using a MHz rate pulse-burst laser system," 35th Aerospace Sciences Meeting and Exhibit. American Institute of Aeronautics and Astronautics, 1997.

[16] Hoffman, D., Münch, K. U., and Leipertz, A. "Two-dimensional temperature determination in sooting flames by filtered Rayleigh scattering," Optics Letters Vol. 21, No. 7, 1996, pp. 525-527.

doi: 10.1364/OL.21.000525

[17] Allison, P. M., McManus, T. A., and Sutton, J. A. "Quantitative fuel vapor/air mixing imaging in droplet/gas regions of an evaporating spray flow using filtered Rayleigh scattering," Optics Letters Vol. 41, No. 6, 2016, pp. 1074-1077. doi: 10.1364/OL.41.001074

[18] Kearney, S. P., Schefer, R. W., Beresh, S. J., and Grasser, T. W. "Temperature imaging in nonpremixed flames by joint filtered Rayleigh and Raman scattering," Applied Optics Vol. 44, No. 9, 2005, pp. 1548-1558. doi: 10.1364/AO.44.001548

[19] Ground, C. R., Gopal, V., and Maddalena, L. "Filtered Rayleigh scattering mixing measurements of merging and non-merging streamwise vortex interactions in supersonic flow," Experiments in Fluids Vol. 59, No. 4, 2018, p. 67. doi: $10.1007 / \mathrm{s} 00348-018-2521-4$

[20] Doll, U., Stockhausen, G., and Willert, C. "Endoscopic filtered Rayleigh scattering for the analysis of ducted gas flows," Experiments in Fluids Vol. 55, No. 3, 2014, p. 1690.

doi: $10.1007 / \mathrm{s} 00348-014-1690-\mathrm{z}$ 
[21] Krishna, Y., Mahuthannan, A. M., Luo, X., Lacoste, D. A., and Magnotti, G. "High-speed filtered Rayleigh scattering thermometry in premixed flames through narrow channels," Combustion and Flame Vol. 225, 2021, pp. 329-339. doi: 10.1016/j.combustflame.2020.10.053

[22] Bergmann, V., Meier, W., Wolff, D., and Stricker, W. "Application of spontaneous Raman and Rayleigh scattering and 2D LIF for the characterization of a turbulent CH4/H2/N2 jet diffusion flame," Applied Physics B Vol. 66, No. 4, 1998, pp. 489-502.

doi: $10.1007 / \mathrm{s} 003400050424$

[23] Krishna, Y., Elbaz, A. M., Yue, Y., and Magnotti, G. "Mole fraction measurement through a transparent quarl burner using filtered Rayleigh scattering," Applied Optics Vol. 58, No. 20, 2019, pp. 5575-5586. doi: 10.1364/AO.58.005575

[24] George, J., and Jenkins, T. P. "Towards Volumetric Measurement of Density using Scanning Sheet Filtered Rayleigh Scattering," 2018 AIAA Aerospace Sciences Meeting. American Institute of Aeronautics and Astronautics, 2018.

[25] Miles, R., Forkey, J., and Lempert, W. "Filtered Rayleigh scattering measurements in supersonic/hypersonic facilities," 28th Joint Propulsion Conference and Exhibit. American Institute of Aeronautics and Astronautics, 1992, pp. AIAA-92-3894.

[26] Miles, R. B., Yalin, A. P., Tang, Z., Zaidi, S. H., and Forkey, J. N. "Flow field imaging through sharp-edged atomic and molecular 'notch' filters," Measurement Science and Technology Vol. 12, No. 4, 2001, pp. 442-451. doi: 10.1088/0957-0233/12/4/308

[27] McManus, T. A., Monje, I. T., and Sutton, J. A. "Experimental assessment of the Tenti S6 model for combustionrelevant gases and filtered Rayleigh scattering applications," Applied Physics B Vol. 125, No. 1, 2019, p. 13. doi: 10.1007/s00340-018-7121-8

[28] Forkey, J. N., Lempert, W. R., and Miles, R. B. "Corrected and calibrated I2 absorption model at frequencydoubled Nd:YAG laser wavelengths," Applied Optics Vol. 36, No. 27, 1997, pp. 6729-6738. doi: 10.1364/AO.36.006729

[29] Müller, D., Pagel, R., Burkert, A., Wagner, V., and Paa, W. "Two-dimensional temperature measurements in particle loaded technical flames by filtered Rayleigh scattering," Applied Optics Vol. 53, No. 9, 2014, pp. 17501758. doi: 10.1364/AO.53.001750

[30] Patton, R. A., and Sutton, J. A. "Seed laser power effects on the spectral purity of Q-switched Nd:YAG lasers and the implications for filtered rayleigh scattering measurements," Applied Physics B Vol. 111, No. 3, 2013, pp. 457-468. doi: $10.1007 / \mathrm{s} 00340-013-5358-9$

[31] Renfro, M. W., Guttenfelder, W. A., King, G. B., and Laurendeau, N. M. "Scalar time-series measurements in turbulent CH4/H2/N2 nonpremixed flames: OH," Combustion and Flame Vol. 123, No. 3, 2000, pp. 389-401. doi: https://doi.org/10.1016/S0010-2180(00)00184-X

[32] Renfro, M. W., Gore, J. P., King, G. B., and Laurendeau, N. M. "Self-Similarity of Hydroxyl-Concentration Temporal Statistics in Turbulent Nonpremixed Jet Flames," AIAA Journal Vol. 38, No. 7, 2000, pp. 1230-1236. doi: $10.2514 / 2.1092$

[33] Sweeney, M. S., Hochgreb, S., Dunn, M. J., and Barlow, R. S. "Multiply conditioned analyses of stratification in highly swirling methane/air flames," Combustion and Flame Vol. 160, No. 2, 2013, pp. 322-334. doi: https://doi.org/10.1016/j.combustflame.2012.10.017

[34] Yang, C., Tang, H., and Magnotti, G. "High-speed 1D Raman analyzer for temperature and major species measurements in a combustion environment," Optics Letters Vol. 45, No. 10, 2020, pp. 2817-2820.

doi: 10.1364/OL.390299 